\title{
The lattice strains in a specimen (hexagonal system) compressed nonhydrostatically in an opposed anvil high pressure setup
}

\author{
Anil K. Singh and C. Balasingh \\ Materials Science Division, National Aerospace Laboratories, Bangalore 560017, India
}

(Received 10 May 1993; accepted for publication 11.January 1994)

\begin{abstract}
A general expression has been derived for the lattice strain which corresponds to the measurement by x-ray diffraction method on a specimen (hexagonal system) compressed nonhydrostatically in an opposed anvil high pressure setup. The expressions relevant lo tlie different diffraction geometries used in high pressurc experiments with opposed anvils emerge as the special cases of this equation. The effect of the deviatotic stress component on tlic lattice parameters derived from the interplanar spacings measured al high pressure is discussed.
\end{abstract}

\section{INTRODUCTION}

The stress state in a solid specimen compressed in an opposed anvil setup deviates from the hydrostatic condition. The effect of nonhydrostaticity on the strains measured by $\mathrm{X}$-ray diffraction methods has been examined by many investigators. A discussion of the earlier work can he found in a recent article, ${ }^{1,2}$ wherein it is shown that the lattice strain. which corresponds to the $x$-ray diffraction measurement, can be calculated only if the anisotropic elasticity theory (AET) is used. The strain in a direction [ $h \mathrm{kl}]$ calculated using isotropic elasticity theory (IET) corresponds to the macroscopic strain which in general differs from the x-ray measured lattice strain in the same direction. For tlic cubic system, the rationf the lattice strain to the macroscopic strain, both along [ $h \boldsymbol{k l}$ ], depends on $(h k l)$ and tlie elastic anisotropy factor $2\left(S_{11}-S_{12}\right) / S_{44}$. The distinction between the two strains vanishes only if the crystallites constituting the specimen are clastically isotropic.

In this article we use AET to derive. for tlic first time, a general expression for the lattice strain produced by the de viatoric stress component in a specimen belonging to hexagonal system compressed nonhydrostatically in an opposed anvil high pressure setup. The expressions for the various diffraction gcomctries used in practice emerge as special cases of this equation. The expression has been derived also for the changes in the lattice parameters caused by the deviatoric stress component. The use of these equations in analyzing the high pressure $\mathrm{x}$-ray diffraction data has been discussed.

\section{if. BACKGROUND INFORMATION}

\section{A. Diffraction geometry}

As discussed earlier, ${ }^{1.2}$ tlie diffraction geometries used in high pressure work fall under tlie following three categories:

(1) Parallel geometry (PLG): This describes the diffraction geometry of a diamorid anvil cell (DAC). The direction of the incident $x$-ray beam coincides with the direction of tie applied load. The angle \$between the diffracting plane normal and the direction of tie applied load equals $(\pi / 2-\theta)$, where $\theta$ is the Bragg angle.

(2) Perpendicular geometry (PDG): This describes the diffraction geometry of tungsten carbide anvil sctup. In this case, $\psi= \pm \pi / 2$.
(3) Scott - Weaver - Takahashi - Bassett - Kinsland (SWEATBAK) geometry: This describes the diffraction ge ometry when DAC is used in the PDG mode. ${ }^{3.4}$ The corn. plete diffraction rings can be recorded on a flat film placed normal to the incident $x$-ray beam. The diffraction ring diameters, parallel and perpendicular to the load axis are of interest, and for these $\psi= \pm \theta$ and $\psi= \pm \pi / 2$, respectively.

\section{B. The stress state}

The stress state at the center of the specimen compressed between tlie anvils will be described with respect to a mutu. ally perpendicular right handed system of coordinates $x_{i}(i)$ $=1,2,3$ ),$x_{3}$ hcing Perpendicular to the anvil face. The origin of coordinates lies midway between the parallel anvil faces on the line passing through the center of and normal to the anvil face. We follow the convention' used in single crystal elasticity theory. As discussed earlier,' the stress at the center of the specimen is given by

$$
\begin{aligned}
\sigma_{i j} & =\left(\begin{array}{ccc}
\sigma_{11} & 0 & 0 \\
0 & \sigma_{11} & 0 \\
0 & 0 & \sigma_{33}
\end{array}\right) \\
& =\left(\begin{array}{ccc}
\sigma_{p} & 0 & 0 \\
0 & \sigma_{p} & 0 \\
0 & 0 & \sigma_{p}
\end{array}\right)+\left(\begin{array}{ccc}
-t / 3 & 0 & 0 \\
0 & & 0 \\
0 & -t / 3 & 0 \\
0 & 2 t / 3
\end{array}\right) \\
& =\sigma_{p}+d_{i j},
\end{aligned}
$$

where $d_{i j}$ is the deviatoric stress component, $\sigma_{p}$ is the mean normal stress, and $I$ is the uniaxial stress component (USC). $\sigma_{p}$, and $t$ are given by

$$
\begin{aligned}
& \sigma_{p}=\left(2 \sigma_{11}+\sigma_{33}\right) / 3=\left(\sigma_{11}+t / 3\right) \\
& t=\left(\sigma_{33}-\sigma_{11}\right) .
\end{aligned}
$$

The maximum shear or von Mises criterion gives

$$
t=\sigma_{y}=2 \tau_{y},
$$

where $\sigma_{y}$ and $\tau_{y}$ denote, respectively, the yield and shear strengths of the specimen material. There exists a radial stress gradient $t^{1,2,6-8}$ in the specimen causing $\sigma_{p}$ and $t$ to vary with the distance from the center. Even though the incidesl 


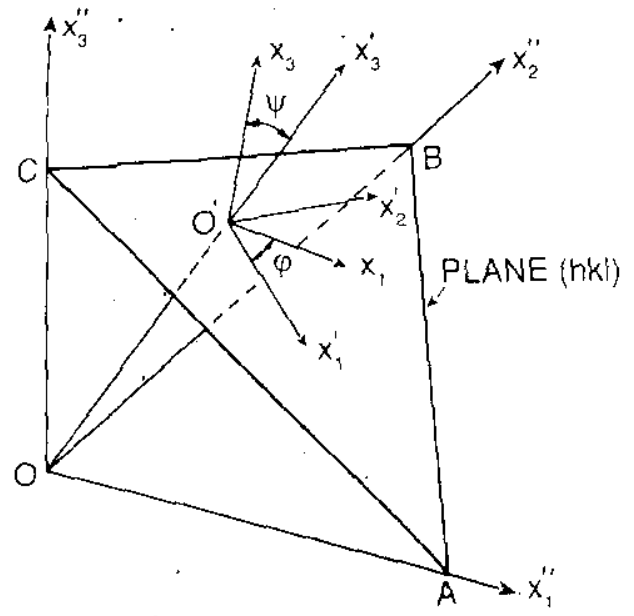

F1G. 1. The relative orientations of the three sets of axes.

$x$-ray beam illuminates a small volume around the center, magnitudes of $\sigma_{p}$ and $t$ vary within this volume. In the following discussion we assume average values of $\sigma_{p}$ and $t$ over the specimen region illuminated by the incident $x$-ray beam.

\section{Choice of axes}

Let us consider a crystallite in the specimen with its plane $(h k l)$ contributing to the intensity at the point of observation. This plane $(A B C)$ referred to the $x_{i}^{\prime \prime}$ axes is shown in Fig. 1. The $x_{1}^{\prime \prime}$ and $x_{3}^{\prime \prime}$ axes coincide with the crystallographic axes $a$ and $c$ of the hexagonal system and $x_{2}^{\prime \prime}$ is chosen perpendicular to both $x_{1}^{\prime \prime}$ and $x_{3}^{\prime \prime}$ to form a right tanded system of coordinates. Another orthogonal set of axes $x_{i}^{\prime}$ is chosen such that $x_{1}^{\prime}$ and $x_{3}^{\prime}$ are along $O^{\prime} A$ and $00^{\prime}$ (normal to the plane $A B C$ ), respectively. $x_{2}^{\prime}$ is in the plane $A B C$. The orientations of $x_{i}^{\prime}$ axes with respect to $x_{i}$ axes are shown in Fig. 1. All the crystallites whose orientations can be obtained by rotating the crystallite about $O O^{\prime}$ contribute to the intensity at the point of observation. These crystallites are termed the $\varphi$ group of crystallites.

\section{EQUATION FOR LATTICE STRAIN}

We first derive the expressions for the lattice strains in a polycrystalline specimen under a stress state given by Eq. (1) under the assumptions of stress continuity (Reuss limit ${ }^{9}$ ) and strain continuity (Voigt limit ${ }^{10}$ ) across the grain boundaries. We then obtain the strain in an actual case as a combination of the strains calculated under the two limits. The expressions for the lattice strains for the hexagonal system have been derived earlier ${ }^{11}$ in the context of $x$-ray elastic constants. Here, we derive the expressions in a self-contained manner, which include the various geometrics used in the high pressure $\mathrm{X}$-ray diffraction work.

The strain component due to $d_{i j}$ in the presence of $\sigma_{p}$ can be calculated by considering $d_{i j}$ acting on the specimen which is already under a hydrostatic pressure $\sigma_{p}$. As the load between the anvils is increased $\sigma_{p}$ increases and the resulting strain can be large; these strains are described best by a stan- dard equation of state. The $d_{i j}$ also increase with increasir $\sigma_{p}$, but remain small as compared with $\sigma_{p}$. For some mat rials, the $d_{i j}$ components may reach a few gigapascals $6 \mathrm{GP}$ at a $\sigma_{p}$ of hundreds of gigapascals. However, since suc materials have large elastic moduli which further increas with increasing $v_{p}$, the strains due to $d_{i j}$ are small. Thus, th use of linear elasticity theory in calculating strains dic to $d$ is justified. The $x$-ray diffraction technique measures inter planar spacings, and therefore, the strain calculated from th measurement at high pressure and 1 atm corresponds to th elastic strain component $\epsilon_{33}^{\prime}$ along $x_{3}^{\prime}$. In the next two sec tions $\epsilon_{33}^{\prime}$ produced by $d_{i j}$ is calculated under the Reuss an Voigt limits.

\section{A. Reuss limit}

We follow essentially the same scheme as used earlier' to derive the expression for $\epsilon_{33}^{\prime}$ arising from $d_{i j}^{\prime \prime}$. The first step is to transform $d_{i j}$ to $d_{i j}^{\prime \prime}$ by two successive tratusformations:

$$
\begin{aligned}
& d_{i j}^{\prime}=a_{i k} a_{j l} d_{k l} \\
& d_{i j}^{\prime \prime}=b_{i k} b_{j l} d_{k l}^{\prime}
\end{aligned}
$$

The single and double prime indicate that the quantity is referred to the $x_{i}^{\prime}$ and $x_{i}^{\prime \prime}$ axes, respectively. The matrices $a_{i j}$ and $b_{i j}$ are given by

$$
a_{i j}=\left[\begin{array}{cccc}
x_{j} & x_{1} & x_{2} & x_{3} \\
x_{i}^{\prime} & & & \\
x_{1}^{\prime} & \cos \varphi \cos \psi & -\sin \varphi & \cos \varphi \sin \psi \\
x_{2}^{\prime} & \sin \varphi \cos \psi & \cos \varphi & \sin \varphi \sin \psi \\
x_{3}^{\prime} & -\sin \psi & 0 & \cos \psi
\end{array}\right]
$$

and

$b_{i j}=\left[\begin{array}{cccc}x_{j}^{\prime} & x_{1}^{\prime} & x_{2}^{\prime} & x_{3}^{\prime} \\ x_{i}^{\prime \prime} & \frac{N}{M} & 0 & \frac{3 c h}{M} \\ x_{1}^{\prime \prime} & \frac{-v^{2} h(h+2 k)}{M N} & \frac{3 a l}{N} & \frac{c(h+2 k)}{M}\end{array} \mid\right.$,

where

$$
\begin{aligned}
& M^{2}=4 c^{2}\left(h^{2}+h k+k^{2}\right)+3 a^{2} l^{2}, \\
& N^{2}=c^{2}(h+2 k)^{2}+3 a^{2} l^{2} .
\end{aligned}
$$

(hkl) are the Miller indices, and a and $c$ the lattice parameters of the hexagonal cell. On carrying out transformations (3) and (4), and averaging over the $\varphi$ group of crystallites 
such that $\left\langle\sin ^{2} \varphi\right\rangle=\left\langle\cos ^{2} \varphi\right\rangle=1 / 2$ arid $(\sin \varphi\rangle=\langle\cos \varphi\rangle=0$ for a completely random orientation of the crystallites in tlic specimen we get

$$
\begin{aligned}
& d_{11}^{\prime \prime}=\left[\frac{1}{2}-\frac{9 c^{2} h^{2}}{2 M^{2}}\right] E, \\
& d_{22}^{\prime \prime}=\left[\frac{1}{2}-\frac{3 c^{2}(h+2 k)^{2}}{2 M^{2}}\right] E, \\
& d_{3,3}^{\prime \prime}=\left[\frac{1}{2}-\frac{9 a^{2} l^{2}}{2 M^{2}}\right] E, \\
& d_{23}^{\prime \prime}=\left[-\frac{3,3 a c l(h+2 k)}{2 M^{2}}\right] E \\
& d_{1,3}^{\prime \prime}=\left[-\frac{9 a c h l}{2 M^{2}}\right] E, \\
& d_{12}^{\prime \prime}=\left[\frac{-3,3 c^{2} h(h+2 k)}{2 M^{2}}\right] E
\end{aligned}
$$

where

$$
E=\left(\frac{1}{3}\right)\left(1-3 \cos ^{2} \psi\right)
$$

The resulting strain $\epsilon_{i j}^{\prime \prime}$ is given by

$$
\epsilon_{i j}^{\prime \prime}=S_{i ; k j} d_{k l}^{\prime \prime} \text {. }
$$

where $S_{i / k l}$ are the clastic compliances in tensor notation. The strain component $\epsilon_{33}^{\prime}$ is given by

$$
\epsilon_{33}^{\prime}=\epsilon_{i j}^{\prime \prime} l_{i} l_{i},
$$

where $l_{i}$ represent the direction cosines of the angles $x_{3}^{\prime}$ makes with $x_{1}^{\prime \prime}$. On carrying out steps (8) and (9) we get

$$
\epsilon_{3,3}^{\prime}=\epsilon_{d}^{R}(h k l)=-\frac{t}{3}\left(1-3 \cos ^{2} / /\right) /\left(2 G_{R}^{x}\right),
$$

where

$$
\begin{aligned}
\left(G_{k}\right) \cdot= & {\left[S_{1,}(1-B)(2-3 B)-S_{12}(1-B)\right.} \\
& -S_{1,3}(1-2 B)(1-3 B)-S_{3,3} B(1-3 B) \\
& \left.+3 S_{44} B(1-B)\right]
\end{aligned}
$$

and

and $S_{m, n}$ are efastic compliances in two-suffix notations at a pressure $\sigma_{p} . \epsilon_{l l}^{R}(h k l)$ represents the strain produced by tlie deviatoric stress component under the Reuss limit. $G_{R}^{x}$ is the slear modulus under the Rcuss limit appropriate to tlic x-ray measurement. It may be noted that $G_{R}^{x}$ is different from the Reuss sheat modutus" $G_{R}$ for the hulk specimen

\section{B. Voigt limit}

The expression for $\epsilon_{33}^{\prime}$ under the Voigt limit can he derived by writing tlic expression for $\epsilon_{3,3}^{\prime}$ for an elastically isotropic case, and substituting for the elastic constants the Voigt-average vilues. This gives

$$
\epsilon_{d}=-\frac{t}{3}\left(1-3 \cos ^{2} \psi\right) /\left(2 G_{V}\right),
$$

wliere $G_{Y}$ is the shear modulus under the Voigt limit gi by"

$$
\left(2 G_{V}\right)^{-1}=X_{1} / X_{2}
$$

wlicrc

$$
\begin{aligned}
X_{1}= & 15 S_{0} S_{44}\left(S_{11}-S_{12}\right), \\
X_{2}= & S_{44}\left(S _ { 1 \mathbf { I } - } S _ { 1 2 1 } \left[2\left(S_{11}+S_{121}+4.513+S_{33}\right]\right.\right. \\
& +6 S_{0}\left[S_{44}+2\left(S_{11}-S_{12}\right)\right], \\
S_{0}= & S_{33}\left(S_{11}+S_{12}\right)-2 S_{13}^{2} .
\end{aligned}
$$

The elastic compliances are at a pressure $\sigma_{p}$. If we ass: that tlie actual stress-strain state in a polycrystalline spi men lies in between those described hy the Reuss and $\mathrm{V}$ limits, then the lattice strain produced by $d_{i j}$ is given by

$$
\Delta \epsilon(h k l)=-\left(\frac{t}{3}\right)\left(1-3 \cos ^{2} \psi\right) /[2\langle G\rangle],
$$

wliere

$$
\langle G\rangle=\alpha G_{R}^{x}+(1-\alpha) G_{V}
$$

and $\alpha$ is a fraction. Obviously $\alpha=1$ and $\alpha=0$ correspono tlic Rcuss and Vnigt limits, respectively. The value of $\alpha$ tc used in an actual case, as discussed in Sec. IV A, is 0.5.

\section{Measured strain}

The strain $\Delta \epsilon(h k l)$ produced by $d_{i}$, in tlie presence large $\sigma_{p}$ is calculated [Eq. (13)] by considering $d_{i j}$ acting the specimen under a hydrostatic pressure $\sigma_{p}$. In terms the measured $d$ spacings. $\Delta \epsilon(h k l)$ is given by

$$
\Delta \epsilon(h k l)=\left[d,,,_{d}(h k l)-d_{p}(h k l)\right] / d_{p}(h k l) .
$$

The subscripts $(p+d)$ and $p$ refer to the measurements un nonhydrostatic pressure $\left(\sigma_{p}+d_{i j}\right)$ and at a hydrostatic $\mathrm{pl}$ surc $\sigma_{p}$, respcctively. The total strain measured under $n$ hydrostatic pressure is given by

$$
\epsilon(h k l)=\left[d_{p+d}(h k l)-d_{0}(h k l)\right] / d_{0}(h k l),
$$

wliere $d_{0}(h k l)$ is the spacings at $1 \mathrm{~atm}$. It is seen from $\mathrm{E}$ (14) and (15) that

$$
\epsilon(h k l)=\epsilon_{p}(h k l)+\left[\mathbf{I}+\epsilon_{p}(h k l)\right] \Delta \epsilon(h k l),
$$
wliere

$$
\boldsymbol{\epsilon}_{p}(h k l)=\left[d_{p}(h k l)-d_{0}(h k l)\right] / d_{0}(h k l) .
$$

In practice the unit cell volume $u$ at various pressure; determined from the measurements at high pressures of th spacings, and the volume strains are calculated. Analogou! the linear (one dimensional) strains defined in Eqs. (1 (171, the following volume strains can be defined:

$$
\begin{aligned}
& \Delta \epsilon(v)=\left(v_{p+d}-v_{p}\right) / v_{p}, \\
& \epsilon(v)=\left(v_{p+d}-v_{0}\right) / v_{0} . \\
& \epsilon_{p}(v)=\left(v_{p}-v_{0}\right) / v_{0} .
\end{aligned}
$$


be subscripts have the same meaning as in Eqs. (14)-(17). From Eqs. (18)-(20) it is seen that

$$
A \bar{\epsilon}(v)=\epsilon(v)-\epsilon_{p}(v)=\left[1+\epsilon_{p}(v)\right] \Delta \epsilon(v) .
$$

\section{DISCUSSION}

\section{A. General}

In an earlier work' the lattice strain in a real situation was expressed as a linear combination of the strains calculated under the Reuss and Voigt limits. Equation (13) in this work makes use of the average of $G_{R}^{x}$ and $G_{V}$, which amounts to expressing the inverse of the strain in a real situalion as the average of the' inverse of the strains calculated under the Reuss and Voigt limits. The two averaging procedures are identical if $G_{R}^{x}=G_{V}$, a case of complete elastic isotropy (see the next section), and give comparable results if $\left(G_{R}^{x} / G_{V}\right)$ does not deviate from unity appreciably. In the tange $0.5<\left(G_{R}^{x} / G_{V}\right)<2$, which covers most real materials, the two calculations differ by less than $11 \%$.A comparison of the two averaging procedures with a rigorous calculation of the strain in a real situation ${ }^{12,13}$ indicates that the average of $G_{R}^{x}$ and $G_{V}(\alpha=0.5)$ provides a better description of strain in a real situation than the linear combination of the strains under the Reuss and Voigt limits when $\left(G_{R}^{x} / G_{V}\right)$ deviates from unity considerably, for example, in the case of zinc for which $\left(G_{R}^{x} / G_{V}\right)$ can reach 0.3 .

\section{B. Elastic isotropy}

A hexagonal system is elastically isotropic if the following conditions are satisfied:

$$
\begin{aligned}
& S_{11}=S_{33}, \\
& S_{44}=2\left(S_{11}-S_{13}\right), \\
& S_{12}=S_{13} .
\end{aligned}
$$

It can be shown that the linear compressibility of a bexagonal system is isotropic if only the first and the last of the tbuve three conditions arc satistied. Thus, the isotropic linear compressibility of a hexagonal system is not a sufficient condition for the complete elastic isotropy.

Under the conditions of complete elastic isotropy, Eq. (13) reduces to

$$
\Delta \epsilon_{\mathrm{is} 0}=-\frac{t}{3}\left(1-3 \cos ^{2} \psi\right) /(2 G)
$$

Under the conditions of isotropy, $G_{V}=G_{R}=\left(S_{44}\right)^{-1}$. Since $G_{V}$ and $G_{R}$ represent, respectively, the upper and the lower bounds" of the shear modulus $G$ of the polycrystidline specimen containing randomly oriented crystallites $\mathrm{G}=\mathrm{G}_{V}=G_{R}$ In terms of the bulk modulus and tlie Poisson's ratio of the specimen material

$$
\Delta \epsilon_{\mathrm{isu}}=-\frac{-}{2}\left(1-3 \cos ^{2} \psi\right)\left|\frac{-}{2 y} \| \frac{-}{2}\right|
$$

where $K$ is the hulk modulus.

\section{Lattice parameters}

The latice parameters $a_{p}+a$ and $c_{p}, d$ determined trom the imerplanar spacings measured at nonhydrostatic high pressure include the contributions from the deviatoric stress component and the hydrostatic pressure component. The changes in the lattice parameters produced by $d_{i j}$ are given by

$$
(\Delta a)_{d}=a_{p+d}-a_{p}
$$

and

$$
(\Delta c)_{d}=c_{p+d}-c_{p}
$$

The suffixes $(p+d)$ and $p$ indicate, respectively, nonhydrostatic and hydrostatic pressure conditions. For $(h k 0)$-iype refections it can bc shown that

$$
d_{p+d}^{-2}(h k 0)=\frac{-}{3} \frac{-}{a^{2}}
$$

and

$$
d_{p}^{-2}(h k 0)=\frac{4}{3} \frac{H^{2}}{a_{p}^{2}},
$$

where

$$
H^{2}=\left(h^{2}+h k+k^{2}\right)
$$

On combining Eqs. (14), (25a), (26a), and (26b), we get

$$
\left(\frac{\Delta a}{a_{p}}\right)_{a}=\Delta \epsilon(h k 0)=-\frac{t}{3}\left(1-3 \cos ^{2} \psi\right) /\left[\langle 2 G\rangle_{h k 0}\right] \text {, }
$$

where

$$
\langle G\rangle_{h k 0}=\frac{1}{2}\left[\left(2 S_{11}-S_{12}-S_{, 3}\right)^{-1}+G_{V}\right] .
$$

By considering (00/)-type reflections and following a similar approach as given above we get

$$
\left(\frac{\Delta c}{\sigma_{p}}\right)_{d}=\Delta \epsilon(00 l)=-\frac{t}{3}\left(1-3 \cos ^{2} \psi\right) /\left[\langle 2 G\rangle_{00 l}\right] \text {, }
$$

where

$$
\langle G\rangle_{001}=\frac{1}{2}\left[\frac{1}{2}\left(S_{33}-S_{13}\right)^{-1}+G_{V}\right] .
$$

For $\cos \psi \leqslant 1 / 33$, both $\left(\Delta a / a_{p}\right)_{d}$ and $\left(\Delta c / c_{p}\right)_{d}$ are positive quantities because a compressive $t$ is negative by convention, and $S_{11}$ and $S_{33}$ are pusitive, and $S_{12}$ and $S_{13}$ arc negativc. Thus, $a_{p+d}>a_{p}$ and $c_{p+d}>c_{p}$. It may be noted that $\Delta \epsilon(h k l)$ for nonzero indices depends on $(h k l)$. When the lattice parameters are obtained hy least squares tit to the measured $d$ spacings, the part of $\Delta \epsilon(h k l)$ for nonzero indices gets absorbed in $a$ and $c$, and the remaining part appears as the scatter in the fit. The numericil values of $a_{p+d}$ and $c_{p+d}$ slightly depend on the type and number of retlecbions used in the calculation. For this reason, the values of $\left(\Delta a / a_{p}\right)_{d}$ and $\left(\Delta c / c_{p}\right)_{d}$ as obtained from Eq. (25a) end Eq. (25b), respectively, will differ slightly from the corresponding values obtained from Eq. (27a) and Eq. (27b). For an isotropic case Eqs. (27a) and (27b) reduce to 
TABLE I. A comparison of the $\left(\Delta a / a_{p}\right)_{d},\left(\Delta c / c_{p}\right)_{d}$, and $\Delta \epsilon(v)$ values for rhenium and cadmium using various equations.

\begin{tabular}{|c|c|c|c|}
\hline $\begin{array}{l}\text { Specimen } \\
\text { material }\end{array}$ & $\left(\Delta a / a_{p}\right)_{a}$ & $\left(\Delta r i c_{p}\right)_{d}$ & $\Delta \epsilon(v)$ \\
\hline \multirow[t]{3}{*}{ Rhenium } & $0.00541^{11}$ & $0.005000^{3}$ & $0.01582^{\mathrm{h}}$ \\
\hline & $0.00 .548^{\circ}$ & $0.00) 4 \times 7^{\text {cl }}$ & $0.015 .580^{\mathrm{t}}$ \\
\hline & $0.0055600^{r}$ & $0.0095600^{\circ}$ & $0.011684^{i}$ \\
\hline \multirow[t]{3}{*}{ Cadmiam } & $0.001 .54^{\circ}$ & $0.00\left(13.35^{a}\right.$ & $0.005 .543^{h}$ \\
\hline & $0.00144^{\circ}$ & $0.000287^{4}$ & $0.00575^{\mathrm{h}}$ \\
\hline & $0.00167^{\circ}$ & $0.000167^{\circ}$ & $0.00501^{1}$ \\
\hline
\end{tabular}

The first six reflections have been used in the last squares fit.

"Calculated from Eq. (29).

"From Eq. (27a).

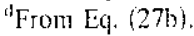

"From Eq. (28).

From Eq. (30)

$$
\left(\frac{\Delta a}{a_{p}}\right)_{d}=\left(\frac{\Delta c}{c_{p}}\right)_{d}=\Delta \epsilon_{\mathrm{is} 1}=-\frac{1}{3}\left(1-3 \cos ^{2}(t) /(2 G) .\right.
$$

The volume strain produced by $d_{i}$ as defined by Eq. (18) is given by

$$
\Delta \epsilon(v)=2(\Delta a / a)_{d}+(\Delta c / c)_{d} .
$$

which for the isotropic case reduces to

$$
\Delta \in\left(v \mathbf{i}=-\left(\frac{1}{3}\right)\left(\mathbf{I}-3 \cos ^{2} \psi\right)(3 / 2 G)\right. \text {. }
$$

Tlic results of Eqs. (27)-(30) wcrc compared with those of tlie least squares tit taken as the benchmark. For this purpose. we used tlie computed $d$ values wliicli simulate the effects of the nonhydrostatic pr ssure. Tlic $d$ values under nonlyydrostatic pressure are given by Eq. (14) which for convenience can he rearranged as follows:

$$
d_{p+d}(h k l)=d_{p}(h k l)[\mathrm{I}+\Delta \epsilon(h k l)] .
$$

The $\mathrm{d}$ values were calculated for rhenium (moderate elastic amisotropy) and cadmium (large elastic anisotropy). The $\mathrm{r}$ values of -6 and $-11.22 \mathrm{GPa}$ for rhenjum and cadmium, respoclively, the dastic compliatnces at $1 \mathrm{~atm}$, and $\alpha=0.5$ were used to compute tlic $d$ values for alj ( $h k l$ ) values listed in Table II. These data correspond to tlic error-free mensurement of the $d$ values under nonhydrostatic pressure with 1 atm mean normal pressure. Since the purpose of this calculation was 10 compare the values of $(\mathrm{Ada},),,, \quad\left(\Delta c / c_{p}\right)_{d}$ and $\Delta \epsilon(v)$ obtained from different equations. no attempt was made to simulate the errors measurement. The $a_{p+d}$ and $c_{p}, d$ values were calculated froin the $d_{p+d}$ values by the method of least squaress. Tlic $\left(\Delta a / a_{p}\right)_{d}$ and $\left(\Delta c / c_{p}\right)_{d}$ wcre calculated using Eqs $(25 \mathrm{a})$ and (25b). respectively; thic values at I atm for $a_{p}$ and $c_{p}$ arc relevant in these calculations. These values along with those calculated using Eqs. (27) and (28) are given in Table $I$. It is seen that in tlie case of rhenium which exhibits moderate alas tic anisotropy, the estimates frorn Eqs. (27) and (28) agree well with those obtained from the least squares lit. In the case of cadmium (with large elastic anisotropy), the estimates of $\left(\Delta a / a_{p}\right)_{d}$ and $\left(\Delta c / c_{p}\right)_{d}$ ob-
TABLE It. The $\boldsymbol{R}$ values with $\alpha=0.5$ for a few elements. Only reffectior with intensity $\geqslant 5$ are considered [intensity of $(101)$ is taken as 100 ].

\begin{tabular}{lcccccccc}
\hline \hline$(h k l)$ & Cd & Co & Hf & Mg & Re & Ti & Zn & Zr \\
\hline 100 & 0.862 & 1.000 & 1.010 & 0.988 & 0.979 & 1.054 & 11.903 & 0.91 \\
002 & 1.311 & 0.770 & 0.923 & 0.919 & 0.853 & 0.871 & 1.342 & 0.85 \\
1011 & 11.1171 & 1.037 & 1.018 & 1.015 & 1.028 & 1.029 & 0.852 & 1.02 \\
1112 & 0.905 & 1.016 & 0.997 & 1.014 & 1.027 & 0.969 & 0.962 & 1.02 \\
110 & 0.862 & 1.000 & 1.010 & 0.988 & 0.979 & 1.054 & 0.903 & 0.97 \\
103 & 1117 & 0.959 & 0.975 & 0.991 & 0.987 & 0.043 & 1.111 & 0.96 \\
112 & 0.883 & 1.040 & 1.013 & 1.018 & 1.034 & 1.022 & 0.855 & 1.03, \\
201 & 0.859 & 1.016 & 1.012 & 0.999 & 0999 & 1.047 & 0.890 & 0.99 \\
211 & 0.860 & 1.010 & 1.011 & 0.995 & 11.491 & 1.050 & 0.889 & 11.98 \\
114 & 1.039 & 0.999 & 0.990 & 1.007 & 1.016 & 0.968 & 1.015 & 1.01 \\
203 & 0.923 & 1.036 & 1.008 & 1.020 & 1.037 & 1.005 & 0.884 & 1.03 \\
\hline \hline
\end{tabular}

tained from Eq. (27) agree wcl] with the corresponding va ucs obtained froni the least squares fit. Equations (28) an (30), which are bised on IET, are not expected to he validfr tlie case of large elastic anisotropy. It is interesting to not however, that the $\mathrm{A} \epsilon(v)$ calculated using the results of $\mathrm{E}$ (30) agrees very well with tlie value, ohtained by the lea! squares tit. The reason for this agreement is discussed in th next section.

\section{AET versus IET}

Equation (23)can he derived " also by assuming that th: bulk specimen is elastically isotropic (as is the case of polycrystalline specimen containing randomly oriented cry! tallites) and using IET to calculate the strain produced by th deviatoric stress component $d$, , As discussed in an earlit article, ${ }^{1,2}$ this strain corresponds to the strain in the bul specimen. and in general, differs from the lattice strai $\Delta \epsilon(h k l)$. This distinction vanishes only if the crystallite constituting the specimen are elastically isotropic. Becaus of their simplicity, the equations derived by IET are ofte used to interpret the experimental data. A comparison of th strains calculated using AET and IET gives an idea of th errors introduced when equations based on IET are used fo a material containing crystallites which are elastically anist tropic. Such a comparison is provided hy tlie ratio $\boldsymbol{R}$,

$$
R=\Delta \epsilon(h k l) / \Delta \epsilon_{\mathrm{iso}}=2 G /\left[G_{R}^{x}+G_{\psi^{\prime}}\right] .
$$

Tlic $R$ values for eight elemental solids crystallizing $\mathrm{i}$ hexagonal system are listed in Table II for various ( $h \mathrm{kl}$ valucs. The elastic compliances ${ }^{\mathrm{t} \sigma}$ and lattice parameters at atm have been used in the calculations. For a given elemen $R$ can he less or more than unity. This means that dependin on $(h \dot{k} l)$, the strain calculated using IET can be larger $c$ smaller than the strain calculated using AET. Thus, whe $\Delta \epsilon(v)$ is calculated by AET using a numher of reflection! tlic differences between AET and IET tend to average ou and as shown in tlie previous section. $\Delta \epsilon(v)$ calculated $h$ IET agrees very well with tlic actual value even for the mi terials showing large elastic anisotropy. Further. for each ement listed in Table II, the effectof anisotropy is maximu for $(00 l)$-type reflections. However, because of preferred ori entation that develops during pressurization, (001)-type re 


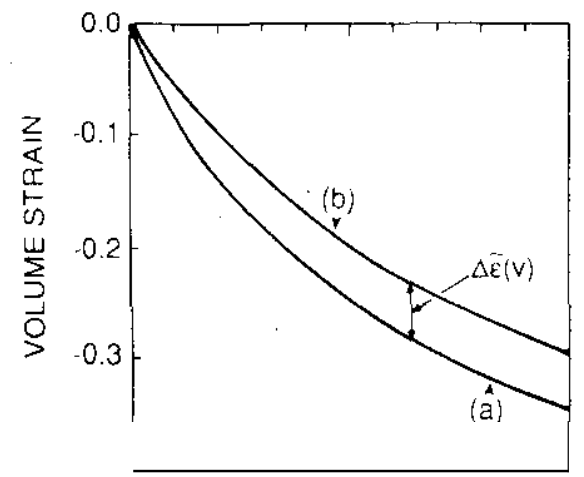

FIC. 2. A typical pv reiation fur a solid. (4) The volune strain $\left(v_{p}-v_{0}\right) / v_{0}$ us pressure $\left(\sigma_{p}\right)$ curve under a hydtostatic condition, (b) The volume strisin $\left(v_{p+d^{-}} v_{0}\right) / v_{0}$ vs pressure $\left(\sigma_{p}\right)$ curve a under nonhydrostatic condition. The offset between (a) and (b) is exaggerated for clarity. $K_{0}$ is the bulk modulus at $1 \mathrm{~atm}$.

Rections are often not observed. The anisotropy effect for other reflections is found to be small. Thus, the assumption of isotropy in the case of the elements listed in Table II does not introduce appreciable error.

\section{E. Estimation of USC}

The estimation of USC $(r)$ from the measurement of the $\mathrm{d}$ spacings under high pressure is of interest, as $t$ characterizes the nonhydrostatic pressure condition and is related to the shear strength of the specimen material [Eq. (2)]. In this section a method of analyzing the $\mathrm{x}$-ray diffraction data to derive $l$ is discussed. This method combines the pressurevolume (pv) relations measured under hydrostatic and nonhydrostatic pressure conditions. A typical pv relation is shown in Fig. 2. The pv curve under nunhydrostatic pressure $\left(\sigma_{p}+d_{i j}\right)$ gives, at each pressure $\sigma_{p}$, a volume strain which is smaller than the corresponding volume strain under hydrostatic pressure $\sigma_{p}$. This is because $\Delta \bar{e}(v)$, the contribution to the volume strain from $d_{i j}$, is positive for $\cos \psi \leqslant 1 / \sqrt{3}$ while $\epsilon_{p}(v)$ is negative. It is to be noted that for the commonly used diffraction geometries for measuring the pv relation, $\cos \psi \leqslant 1 / \sqrt{3}$. If the ruby fluorescence technique is used to measure the pressure under a nonhydrostatic condition, then the measured pressure corresponds ${ }^{\mathrm{t} 7}$ to $\sigma_{p}$, The pressure estimated by mixing a material (pressure marker) of known pv relation with the specimen and measuring the volume strain, corresponds to $\sigma_{p}$ only 'if the shear strength of the marker material is low as compared with that of the specimen material. However, if the shear strengths of the marker and specimen materials are comparable, then the estimated pressure is not simply related to $\sigma_{p}$. Assuming that the pressure measused in the experiments under the nonhydrostatic pressure "conditions is $\sigma_{p}$, the difference between the volume strains,
$\Delta \bar{\epsilon}(v)$, under nonhydrostatic and hydrostatic pressure conditions is given by Eq. (21). On combining Eqs. (21), (27a), (27b), and (29) we get

$$
\begin{aligned}
t= & -\frac{3 \Delta \tilde{\epsilon}(v)}{\left(1-3\left\langle\cos ^{2} \psi\right\rangle\right)\left[1+\epsilon_{p}(v)\right]} \\
& \times\left[\frac{2}{\langle 2 G\rangle_{h k 0}}+\frac{1}{\langle 2 G\rangle_{00 t}}\right]^{-l},
\end{aligned}
$$

$+2$

$$
t=-\frac{\Delta \tilde{\epsilon}(v)(3 K)}{\left(1-3\left\langle\cos ^{2} \psi\right\rangle\right)\left[1+\epsilon_{p}(v)\right]}\left(\frac{1-2 v}{1+\nu}\right) .
$$

It may be noted that the elastic constants in Eqs. (32) and (33) are at a pressure $\sigma_{p}$. At a given pressure, the $\Delta \bar{\theta}(v)$ can be obtained from the strain offset between the pv relations under nonhydrostatic and hydrostatic pressure conditions. Often, it is not possible to measure the pv relation under a hydrostatic condition in the megabat pressure range. In such cases, the pv relation under the hydrostatic pressure condition can be computed using high precision measurements of the bulk modulus and its pressure derivative. and a suitable theoretical equation of state. ${ }^{20}$ The use of Eq. (32) requires the elastic compljances at a pressure $v_{p}$, which can be obtained if the etastic compliances, their pressure derivatives, and a suitable method of extrapolating these to high pressure are available. However, Eq. (33) derived under the condition of elastic isotropy requires $K$ and $\nu$ at $\sigma_{p} . K$ at high pressure can be obtained from the value of $K$ and its pressure derivative at $1 \mathrm{~atm}$, and a theoretical equation of state. The pressure dependknce of $v$ is often small and a linear extrapolation to high pressure is adequate in most cases. As the assumption of elastic isotropy in a hexagonal system. does not introduce appreciable error, $\mathrm{Eq}$. (33) can be used conveniently in calculating I from measured $\Delta \tilde{\epsilon}(v)$.

The SWEATBAK geometry permits the recording of the complete diffraction ring on a flat film placed normal to the incident x-ray beam. The strains $\epsilon_{g \eta}(h k l)$ and $\epsilon_{\perp}(h k l)$ can be calculated using Eq. (16) and the diffraction ring diameters measured parallel and perpendicular to the load axis, respectively. Using Eqs. (16) and (23)we get the following relation for the elastically isotropic case:

$$
t=\frac{3 K\left[\epsilon_{\theta}(h k l)-\epsilon_{\perp}(h k l)\right]}{\left[1+\epsilon_{p}(h k l)\right] \cos ^{2} \theta}\left(\frac{1-2 \nu}{1+\nu}\right) .
$$

\section{F. Effect of preferred orientation}

A completely random orientation of the crystallites in the specimen has been assumed in the present analysis such that $(\cos \varphi\rangle=\langle\sin \varphi\rangle=0$ and $\left\langle\cos ^{2} \varphi\right\rangle=\left\langle\sin ^{2} \varphi\right\rangle=1 / 2$. However, specimens of hexagonal close packed metals upon compression between the anvils are known to exhibit preferred ori- 
entation (texture). ${ }^{20}$ In such cases, the average values of the trigonometric functions will have to be evaluated using expressions given earlier.' This will require complete knowledge of the crystallite orientation distribution function in the region of the specimen illuminated by the $\mathrm{x}$-ray beam during the high pressure experiments.

\section{CONCLUSIONS}

General expressions have been derived for the lattice strains in the specimen belonging to the hexagonal system compressed nonhydrostatically in an opposed anvil setup. The expressions for the various diffraction geometries used in high pressure work emerge as special cases of these equations. The use of these equations in practice to analyze the $\mathrm{x}$-ray diffraction data requires knowledge. of the elastic compliances at high pressures. Assumption of elastic isotropy of the crystallites comprising the specimen introduces considerable simplification in the equations. The strains calculated by AET and IET for any given $(\boldsymbol{h} \boldsymbol{k} \boldsymbol{l})$ are found to differ by 'less than $15 \%$ for most elements in hexagonal system. Even for highly anisotropic materials such as cadmium, cobalt, and zinc, a large anisotropy effect is observed for only (001)-type reflections. The equations derived using IET can be used to analyze the diffraction data from hexagonal systems without introducing appreciable error.
'A. K. Singh, J. Appl. Phys. 73, 4278 (1993).

'A. K.Singh, I. Appl. Phys. 74, 5920 (1993).

${ }^{3}$ J. \$ Weaver, T. Takahashi, and W. A. Bassett, Eos Trans. Am. Geophys. Union 53,511(1972)

G. L. Kinşland and W. A. Bassett, Rev. Sci. Instrum. 41, 130 (1976).

${ }^{5} J$. F. Nve, Physical Properties of Crystals (Oxford Unjyersity Press. Lon. don, UK, 1960l.

${ }^{6} \mathrm{C},-$ M. Sung, C. Goetze, and H. K. Mao, Rev. Sci. Instrum. 48, 1386 (1977).

'H. Kimura, D. G. Ast, and W. A. Bassett, J. Appl. Phys. 53, 3253 (1582). ${ }^{8} \mathrm{C}$. Mead and R. Jeanloz, J. Gcophys. Res. 93, 3261 (1991).

${ }^{9}$ A. Reuss, Z. Angew. Math. Mech, 9, 49 (1929).

${ }^{10}$ W. Voigt, Lehrbuch der Kristallphysik (Toubner, Leipzig, 1928).

${ }^{11}$ P. D. Everschor, W. Froiich. and V. Hauk, Z Metallk, 62, 38 (1971).

${ }^{12}$ E. Kroner, Z. Phys. 151,504 (1958).

"P.D. Evensehor and V. Hauk, Z. Motallk, 63, 798 (1972)

${ }^{14}$ R. Hill, Proc, Phys. Soc. (London) A 65, 349 (1952).

"A. L. Ruoff, H. Lua, H. Xia, and Y, K. Vohra, High Press. Ros, 6, 183 (1991).

${ }^{16} \mathrm{G}$. Simmons and H. Wang, Single Crysfol Elastic Constants and Calcu. lated Aggregate Properties: A Handbook (MTT Cambridge. MA, 19711.

17 It may be noted that under a nonhyorostatic condition, the pressure estimated from the shifts of the $\mathrm{R}$ lites depends slightly on the crystallo. graphic orientation of the ruby chip with respect to the $x_{;}$axes (Ref. 17) The pressure determined using the $R_{2}$ line is a better indicator of $\sigma_{\mathrm{p}}$ as the response of the $R_{2}$ line to stross is less anisotropic (Refs. 17 and 18). Howover, the $\mathrm{R}$ lines are often not well resolved at high nonhydrostatie pressures.

${ }^{18} \mathrm{~S}$. M. Sharma and Y. M. Gupla, in Recent Trends in High Pressure Re. search, edited hy A. K. Singh (Oxford and IBH, New De] hi, 1992), p. 7

${ }^{19}$ Y. M. Gopta and X. A. Sten, Appl. Phys. Lott. 58, 583 (1991).

${ }^{20} \mathrm{R}$ Jean[oz, B. K Godwal, and C.. Mcade. Natuse 349. 687 (1995). 